\title{
Introduction of Life Cycle Management to Organisations. LCA And Other Supporting Tools
}

\author{
Zbigniew Klos
}

The idea of assistance of different tools in introduction of Life Cycle Management of products and services to the organizations in Poland and other Central and Eastern European countries is presented in this paper. First, Total Quality Management, ISO 9001 and ISO 14001 standards are analyzed as a tools which could support this process, but special attention is paid to Life Cycle Assessment (LCA). According to environmental management systems scheme organisations of all kinds are concerned to achieve and demonstrate the sound environmental performance and they have to establish and maintain the procedures for examining and assessing the environmental effects of their activities, products or services. Among the best in this field methods is just LCA. It is the process of evaluating the environmental effects by identifying and quantifying the environmental loads or interventions, i. e. the amount of energy and raw materials used and the amount of emissions and wastes released into the environment. In this paper the goals of LCA are listed and main applications are presented.

\section{Introduction}

Billions of technical objects produced, used and then disposed in the world have the great influence on different areas: economy, safety and environment. Specially the last one is recently of great interest to many organizations worldwide. They undertake various efforts to reduce the impact of their products or services on environment. Some of them try to do it in broader sense, introducing Life Cycle Management (LCM). This practical approach is focused on minimising the environmental burdens associated with products and services over their entire „life” through better, environmentally oriented, decision making. It is started to be implemented in modern companies, with the different structure and content.

Polish economy is still on its way from centrally-steered to market-oriented. Free market rules have had to be accepted, but with fulfilment of several different regulations, amongs them of environmental character, there are still problems. Although LCM is up to now rather the concept then consequently structured regulation it has the chance to be formed in the future as a integrated tool. It should attract the better developed companies even in Poland and other Central and Eastern European (CEE) countries. However some supporting tools should be use to make this process more efficient.

\section{Total Quality Management}

Now, at the beginning of new century, some Polish quality experts state that an introduction of Total Management Quality (TQM) is one of the last chances for the Polish economy to revitalize it. It is obvious in the situation of the entry of Poland into global market now and joining the European Union soon. Our country and neighbour countries have lately undergone transformation, but the necessity of adaptable works which still should be done is evident. The best methods in this field are originated from TQM and culture area. They could be applied at the different levels of economy, being specially suitable in the companies (organisations).

Considering the basis for development now LCM systems in CEE countries it seems that the attention should be focused first on introducing in organizations the rules of TQM and the regulations of such a standards as ISO 9001 and ISO 14001. Some of parts of these standards include elements which could support introduction of LCM and they will be presented later in this paper.

TQM could be defined as the way of organisation management, characterised by: system approach, orientation on strategic goals, ability to continuous and for ever improvement and active team activity of whole staff with full engagement of top management, which leads to fulfil the needs of customers in given moment and in the future with the minimum costs, done by use of people and applied by them quantitative methods to: assessment and rationalisation of all important processes in the organisation and improvement of delivery (materials and services) supplied to the organisation [1].

First one - system approach - is the activity, which uses the ordered set of mutually interrelated elements of given entirety for its maximum effectiveness. The purpose of the creation of such an entirety is the long-term profit for all interested parties of given community. Defined in this way system approach does not exist in and around the majority of Polish organisations. Individual examples are the exceptions. It is rather seen opposite tendency in organisations to confine their frames to minimum dimensions. Besides the positive role of cutting superfluous units, this process brings the threat of liquidation of departments which are extemporary non-profit, but needed for long-term operation of organisation, for example $R \& D$ departments.

In survey done by B. Nogalski, $75 \%$ of organisations declared that they had their own strategy, but over $70 \%$ 
of them had only short-term ones [2]. Thanks to the TQM, the goals of the organisations are maximised and the specific consonance and stability of goals are created. As it is seen in Poland, there are only few results of such an activity. The goals of organisations rarely go down to departments and to the single staff member.

Ability for continuous and for ever improvement is known, new philosophy of systematic process improvement of its functioning ought to be build-in, instead of situation of fighting fires or quick fixes. It is obvious, that it is much easier to do it in stable surroundings of constant rules of market. Considering still changeable principles of operation on the Polish market, only few organisations have the good possibilities in respect with it.

The greatest challenge in the whole chain of activities in introduction of TQM to organisations is probably active team engagement of full staff to work on improvement of quality. The consciousness of the importance of quality factor for organisation market success is still limited, specially amongst workers. Top management is deeply influenced by some factors, also the threat of job loss. It has been stated that in Poland two times more persons from top management reach the satisfaction from their work then workers.

The fulfilment of customer needs should be reached with minimal costs. The production processes are still often organised in the way to minimise first of all the costs. The quality level is only the derivative of this fact. Changes connected with privatisation increase the range of the application of economical tools of management, mainly for minimisation of costs.

Use of quantitative method and people to rationalisation of all important processes in organisation is various. In many of them the application of quality assurance tools stopped at the level of SPC. The importance of described here aspect is visible, when one consider the hierarchy of sources of work effectiveness. The need of processes rationalisation is here on the first position.

Cultural change is not often met in many organisations because the culture, as a system of common values and goals, is mainly the stable element of organisations. In many companies the endeavour to reach first of all the quantitative goals, specially financial, is very strong. Improvements, which are not leading to above mentioned goals, are not popular. As an element of desired culture change, the need of continuous rationalisation of organisation structure is seen. This idea, specially for staff of state-owned organisations, is still often new.

There are six main steps in TQM implementation process:

- understanding,

- top management commitment,

- company-wide awareness,

- planning,

- implementation,

- review, leading to increased understanding and continuous improvement.
Implementation is divided into categories of people, technology and structure [3]. Although the matter is different, the structure of LCM implementation is very similar, which helps to reach positive results.

\section{Cultural factors}

The term "enterprise (or company or organisation) culture" is more commonly known only from about thirty years. It is defined that the corporate culture is the pattern of declared convictions and values, which give the corporate co-workers the sense of activities and rules of behaviour [4]. Such culture symptoms are seen:

- criteria of promoting to higher ranks,

- criteria of recitation,

- way of contacts with customers,

- layout and the way of creation of organisation documentary,

- architecture and the state of organisation infrastructure,

- readiness of co-workers to work in overtime,

- way of communication in organisation.

Factors which are connected with culture introduction to Polish companies can be gathered in two groups:

1) external, dependent on factors from the environment of corporate,

2) internal, typically inside corporate dependent.

Speaking about the external factors influencing the introduction of culture to Polish enterprises one can remember about two aspects: historical conditions, which shape the situation in Poland not only in economy, but also in social area, and actual ones.

All facts which have influenced on the attitude towards work, its quality and the quality of products have had historical conditions. Among the actual conditions one can distinguish:

- economical,

- social.

As a actual key condition for successful introduction of culture strategy to Polish organisations, the reconstruction of the whole national economy for the qualityoriented goals is mentioned. S. Sudol presenting the results of the inquiry shows, that the factors which are friendly to reaching these goals, the experts arranged in following order [5]:

c) socially accepted, concise pro-quality concept of economy,

d) complete turnover from centrally-steered to marketcontrolled economy,

e) significant increase of foreign investments,

f) consequence in introduction of market-controlled economy,

g) demonopolization of production,

h) remarkable structural changes in Polish economy.

The demand of acceptance and introduction of proquality concept of economy $(\mathrm{a}, \mathrm{d}, \mathrm{f})$ is more important 
then the changes of management mechanisms (b, e) and inflow of foreign capital (c), as we see from above.

Speaking about the social conditions one can mentioned the specific national features of Poles, which belong also to the external, from the point of view of position to organisation, conditions. They are, as follows: skill of achieving the goals in unconventional ways, unrecognition of authorities, aspiration to freedom and need of quick personal success. Poles are seen world-wide as an individualists with high ambitions, open-minded, flexible, talented improvisers, innovative and fast learning. When one combine these features with the Polish predilection to improve everything around and the great criticism, this mixture is seen by E. Kindlarski to be promising starting point for attaining quality goals [6].

\section{ISO 9001 standard}

The International Standard ISO 9001 [7] is one of three international standards dealing with quality system requirements that can be used for external quality assurance purposes. The quality assurance model is there the broadest and acompasses: design, development, production, installation and servicing. There are several elements of this model which include the activities influencing the environment:

1) management responsibility - managers shall define and document their policy for quality, which shall be relevant to the expectations and needs of customers (initiation of actions to prevent the occurrence of any nonconformities related to the product, process and quality system, review of quality system at defined intervals sufficient to ensure its continuing suitability and effectiveness in satisfying the requirements),

2) design control - establishing and maintaining of documented procedures to control and verify the design of the product in order to ensure that the specified requirements are met (design input requirements relating to the product, including applicable statutory and regulatory requirements shall be identified, documented and their selection reviewed for adequacy with safety and proper functioning of the product),

3) purchasing - establishing and maintaining the procedures ensuring that the purchased product conforms to specific requirements (the supplier shall evaluate and select subcontractors on the basis of their ability to meet subcontract requirements including the quality system and any specific quality assurance requirements),

4) process control - identification and planning the production, installation and servicing processes which directly affect quality and shall ensure that these processes are carried out under controlled conditions (documented procedures defining the manner of production, installation and servicing, criteria for workmanship, which shall be stipulated in the clearest practical manner, etc.)

5) inspection and testing - establishing and maintaining of documented procedures for inspection and testing activities in order to verify that the specified requirements for the product are met (the supplier shall ensure that incoming product is not processed until it has been inspected or otherwise verified as conforming to specified requirements, in accordance with the quality plan and/or documented procedures; the supplier shall carry out all final inspection and testing in accordance with the quality plan and/or documented procedures to complete the evidence of conformance of finished product to the specified requirements),

6) corrective and preventive action - establishing and maintaining documented procedures for implementing corrective and preventive action (the range of action shall be to a degree appropriate to the magnitude of problems and commensurate with the risks encountered),

7) auditing - establishing and maintaining documented procedures for planning and implementing auditing to verify whether activities comply with planned arrangements,

8) servicing - establishing and maintaining documented procedures for performing, verifying and reporting that the servicing meets the specified requirements [7].

\section{ISO 14001 standard}

In the context of increasingly stringent legislation, the development of economic policies and other measures to foster environmental protection, and a general growth of concern from interested parties about environmental matters, including sustainable development, organisations of all kinds are concerned to achieve and demonstrate sound environmental performance, by controlling the impact of their activities, products and services on environment, taking into account their policy and objectives.

International standards covering environmental management are intended to provide organisations with the elements of an effective environmental management system which can be integrated with other management requirements, to assist organisations to achieve environmental and economic goals. The ISO 14001 [8] standard specifies the requirements of such an environmental system, which could be applicable to all types and sizes of organisations and to accommodate diverse geographical, cultural and social conditions. A system of this kind enables an organisation to establish and assess the effectiveness of procedures to set an environmental policy and objectives, achieve conformance with them, and demonstrate such conformance to others. The overall aim of this standard is to support environmental protection and prevention of pollution in balance with socio-economic needs. Many of the requirements may be addressed concurrently or revisited at any time.

The adoption and implementation of a range of environmental management techniques in a systematic manner can contribute to optimal outcomes for all interested 
parties. However, adoption of this standard will not in itself guarantee optimal environmental outcomes. In order to achieve environmental objectives, the environmental management system should encourage organisations to consider implementation of the best available technology, where appropriate and where economically viable.

The ISO 14001 standard shares common management system principles with the ISO 9000 series standards. Organisations may elect to use an existing management system consistent with the ISO 9000 series as a basis for its environmental management system. The application of various elements of management system may differ due to different purposes and different interested parties. While quality management systems deal with customer needs, environmental management systems address the needs of a broad range of interested parties and evolving needs of society for environmental protection. Here it has the strong common point with LCM.

In the ISO 14001 standard requirements for an environmental management system are specified, to enable an organisation to formulate policy and objectives taking into account legislative requirements and information about significant environmental impacts. It applies to those environmental aspects which the organisation can control and over which it can be expected to have an influence.

The organisation shall establish and maintain an environmental management system, the requirements of which are in following fields:

1) environmental policy - top management shall define the organisation's environmental policy and ensure that it includes a commitment to comply with relevant environmental legislation and regulations, and with other requirements to which the organisation subscribes and it is appropriate to the nature, scale and environmental impacts of its activities, products and services,

2) objectives and targets - environmental objectives and targets, at each relevant function and level within the organisation should be established and maintained, considering the legal and other requirements, significant environmental aspects, technological options and financial, operational and business requirements,

3) planning - procedure should be established and maintained to identify the environmental aspects of the activities, products or services that it can control and over which it can be expected to have and influence, in order to determine those which have or can have significant impacts on the environment ( aspects related to significant impacts should be considered in setting environmental objectives),

4) legal and other requirements - procedure should be established and maintained to identify and have access to legal and other requirements to which organisation subscribes, that are applicable to the environmental aspects of its activities, products or services,
5) environmental management programmes - should be established and maintained for achieving objectives and goals,

6) implementation and operation - management shall provide resources (human, financial and technology resources) essential to the implementation and control of the environmental management system,

7) operational control - organisation shall identify those operations and activities that are associated with the identified significant environmental aspects in line with its policy, objectives and targets,

8 ) emergency preparedness and response - procedures should be established and maintained to identify potential for and respond to accidents and emergency situations, and for preventing and mitigating the environmental impacts that may be associated with them,

9) monitoring and measurement - procedures should be established and maintained to monitor and measure, on regular basis, the key characteristics of operations and activities in organisation that can have a significant impact on environment [8].

\section{Methodology of an Life Cycle Assessment}

\subsection{General remarks}

Most products require a large variety of production, distribution, use and disposal processes. Each process can produce a large variety of emissions and each emission has a very specific effect on the environment.

The complex interaction between a product and the environment is dealt with in a Life Cycle Assessment (LCA) method. In the above ISO 14040 standard [9], LCA is defined as follows:

"Life Cycle Assessment is a technique for assessing the environmental aspects and potential impacts associated with a product, by:

- compiling an inventory of relevant inputs and outputs of a system,

- evaluating the potential environmental impacts associated with those inputs and outputs,

- interpreting the results of the inventory and impact phases in relation to the objectives of the study."

This framework consist of the following phases of LCA methodology [10]:

- goal and scope definition, in which amongst others the goal and scope of the study are specified, the functional unit of the study is defined and a quality insurance procedure established,

- inventory analysis, in which all relevant inputs from, and outputs to, the environment are specified and, if possible, quantified for entire life cycle of the product,

- impact assessment, in which the potential environmental impacts are assessed for these inputs from, and outputs to, the environment,

- interpretation, in which sensitivity analyses are performed and a general appraisal of the results made in relation to the defined goal of the study (Figure 1). 


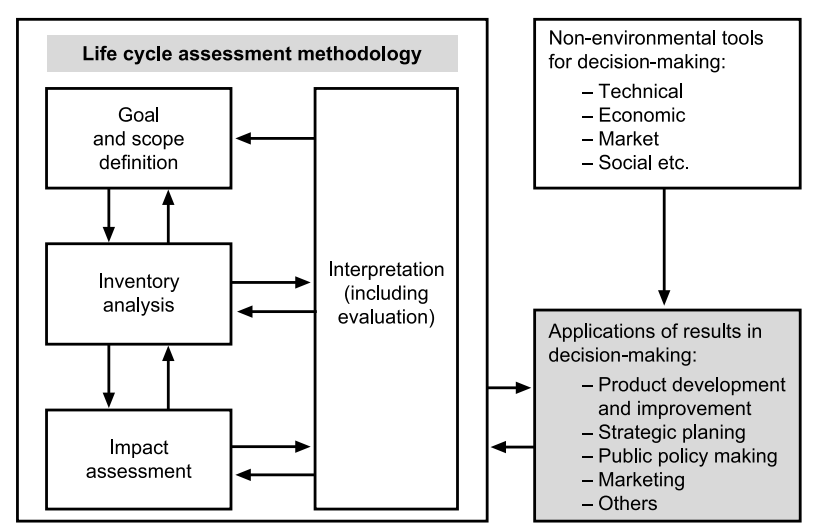

Figure 1. Phases of LCA-arrows indicating logical flow of information [11]

\subsection{Goal and scope definition}

The goal and scope definition phase consists of at least the following elements [10]:

- specification of the purpose and scope of the study,

- definition of the functional unit,

- establishment of the level of detail required for the application hand,

- a procedure for ensuring the quality of the study.

The definition of the purpose of the life cycle assessment is an important part of the goal definition. The practitioner, who has to reach the goal, needs to understand the detailed purpose of the study in order to make proper decisions throughout the study. Examples of goals of a life cycle assessment are [12]:

- to compare two or more different products fulfilling the same function with the purpose of using the information in marketing of the products or regulating the use of the products,

- to identify improvement possibilities in further development of existing products or in innovation and design of new products,

- to identify areas, steps etc. In the life cycle of a product where criteria can be set up as part of the ecolabelling criteria to be used by e. g. the ecolabelling board.

\subsection{Inventory analysis}

The second phase is the inventory analysis - the core phase of LCA and, in fact, the most objective part of the study. It is also the most time consuming phase and it consists of at least following elements [13]:

- drawing up a flow chart of the processes involved (Figure 2),

- definition of the boundaries between product system and environment,

- specification of processes and data gathering,

- allocation regarding multiple processes,

- compilation of the inventory table.

The inventory analysis includes collection and treatment of data to be used in preparation of a material consumption, waste and emission profile for all the phases in the life cycle, but also for the whole life cycle.
The inventory table is an environmental accounting balance sheet containing the burdens identified for each of the separate activities that are included within the system and show the inputs and outputs from the system in their entirety in terms of the resources required by the system and the emission released during production, transportation, use and disposal.

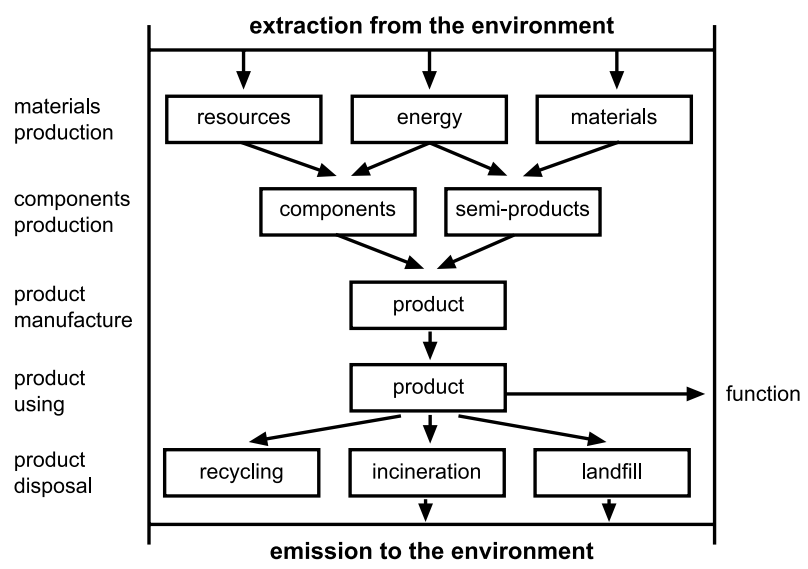

Figure 2. Example of process flowchart [13]

\subsection{Impact assessment}

Impact assessment is the third phase in a life cycle assessment containing the following main issues: classification, characterisation, normalisation and valuation. A brief description of these terms is provided below.

Environmental burdens arise as a result of the materials and energy flows, process activities, use of land and waste handling. Commencing with the inventory data for the system under consideration, the classification stage assigns each environmental burden within the inventory table to one or more impact categories [13]. For example, environmental burdens resulting from traffic movements will include emissions of combustion gases which could contribute towards global warming or human health effects. Methane emissions form landfills are linked with both photochemical oxidant formation and global warming, while sulphur dioxide emissions from incinerators are associated with both human toxicity and acidification. Impact associated with steel production could include resource depletion and ecotoxicological impacts.

In the characterisation stage, the relative contribution of each burden, on a mass basis, to any one impact category is assessed by giving each burden a weighting factor which may then be used as a multiplication factor for each burden. The factors are assigned relative to a reference burden. The product of the weighting factor and the mass burden for a particular substance is termed its "score". The total score in each impact category is therefore the sum of the individual burdens.

The purpose of normalisation is to relate the data from the characterisation stage to the total magnitude of the given impact category in some given area and time [14]. This may be achieved by dividing the total score 
obtained within each category by some area or time. For example, all global warming species scores are typically expressed in terms of their $\mathrm{CO}_{2}$ equivalents on a global level, over, for instance, a 100 year time frame. Normalisation can in some cases provide a better basis for valuation and interpretation of impact data.

The relative importance of each of the different potential environmental impacts are weighted against each other in valuation stage. This stage may involve both qualitative and quantitative valuations - political and ethical values may need to be invoked. Hence, this stage is essentially normative. Classification and characterisation are considered to be the more generally scientific stages, whereas valuation includes subjective judgements and is often regarded as a separate exercise.

\subsection{Interpretation}

Interpretation is the fourth phase in life cycle assessment containing the following main steps [12]:

- identify the significant environmental issues,

- evaluate the methodology and results for completeness, sensivity and consistency,

- check that conclusions are consistent with the requirements of the goal and scope of the study,

- final conclusions.

\subsection{Application of LCA}

Main fields of LCA applications are, as follows:

a) Internal industrial use in product development and improvement - LCA can be used at an operational level for product improvement, product design and product comparison. The latter is, for instance, at stake in the underpinning of ecolabelling programs or purchase schemes;

b) Internal strategic planning and policy decision support in industry - LCA can be used at a strategic level, either by companies or authorities. Within firms, LCA may give the guidance for business strategies, including decisions as to which types of products to develop, which types of resources to purchase and which type of investments to make for waste management. For authorities, an application of increasing importance is the comparison of contrasting environmental policy options, for instance, in the fields of waste management, energy policy or transportation;

c) External industrial use for marketing purposes;

d) Governmental policy making in the areas of ecolabelling, green procurement and waste management opportunities;

e) LCA can be used as a screening tool, identifying key issues for further investigation by other means.

\section{Conclusions}

Polish economy, after being one of the fastest growing economies in Europe, is now in crisis. Having in mind this situation, Polish organisations should, and I hope that they will, work with increasing force on improving their quality and environmental systems. Also official confirmation of quality system existence, by appreciating for certificate, will be of interest for thousands of organisations. Number of several thousands of ISO 9000 certificates, obtained in last years, will increase now in faster rate.

It is to observe among the organisations, which are ISO 9000 and ISO 14000 certificate holders for some years, that they try to reach now higher level of excellence in quality area by introducing TQM and shaping their own individual corporate culture. I hope that this sound tendency will be kept in future including also LCM.

It is worth to mention that the problem of LCM implementation in small and medium enterprices (SME) is of key importance because they constitute in many countries (e. g. Western Europe) almost 2/3 of private sector and represent a major source of growth in employment, through new business start-ups. Thus the SME sector is an important target for competitiveness, innovation and environmental policies and practices but reveals low awareness of environmental issues. Results from research show that most SMEs:

- do not consider that their operations have an impact on the environment,

- are umfamiliar with environmental systems, respond to regulation as the biggest stimulus for introducing environmental measures,

- are unware that they have a legal Duty of Care to ensure that their wastes are handled and disposed of properly [15].

\section{References}

[1] Klos Z., Introduction of TQM strategy to Polish enterprises - analysis of conditions. Organizacja i Kierowanie, 1996, no 1

[2] Nogalski B., Privatisation - symptoms of changes. Przeglad Organizacji, 1993, no 3

[3] Cullen J., Hollingum J., Implementing TQ, IFS Ltd., Springer Verlag, Berlin 1987

[4] Davies S. M., Managing corporate culture. Harper \& Row Publ. New York 1985

[5] Sudol S., Pro-quality policy. Przeglad Organizacji, 1992, no 2

[6] Kindlarski E., Polish Quality Way. Proceedings of the 36th Quality Congress, Brussels 1992

[7] ISO 9001 Quality systems - Model for quality assurance in design, development, production, installation and servicing

[8] ISO 14001 Environmental management systems - Specification with guidance for use

[9] ISO 14 040: Environmental Management, Life cycle assessment, Principles and framework

[10] Udo de Haes H. A., E. van der Voet, Material Flow Accounting and Life Cycle Assessment. The Global Environment. 
Science, Technology and Management, Leiden Scandinavian Science Publishers 1997, s. 1151-1171

[11] Huppes G., LCA yesterday, today and tomorrow, w: RITE International Workshop about Total Ecobalance, February 1996, s. 29-51

[12] Jensen A. A. et al., Life Cycle Assessment (LCA), A guide to approaches, experiences and information sources, Report to the European Environment Agency, dk-TEKNIK Soborg 1997

[13] Fava J. A., Consoli F., A technical Framework for Life-Cycle Assessment. Workshop report, Washington, SETAC USA 1993

[14] Consoli F. et al., Guidelines for Life Cycle Assessment: A Code of Practice. Brussels, Society of Environmental Toxicology and Chemistry (SETAC), 1993, Belgium

[15] Bradford D, Motivating SMEs towards improved environmental performance. The IPTS Report, 2000, vol. 41

\section{Author}

Prof. Dr. habil. (Eng.) Zbigniew Klos

Poznan University of Technology

Institute of Machines and Motor Vehicles

ul. Piotrowo 3, 60-965 Poznan, Poland

Tel. +48 (61) 6652231

Fax +48 (61) 6652736

E-Mail: zbigniew.klos@put.poznan.pl 